\title{
Auxin-BR Interaction Regulates Plant Growth and Development
}

\author{
Huiyu Tian ${ }^{\star}$, Bingsheng Lv ${ }^{\dagger}$, Tingting Ding, Mingyi Bai* and Zhaojun Ding* \\ The Key Laboratory of Plant Cell Engineering and Germplasm Innovation, Ministry of Education, College of Life Sciences, \\ Shandong University, Jinan, China
}

OPEN ACCESS

Edited by:

QiXie,

Institute of Genetics and Developmental Biology (CAS),

China

Reviewed by:

Elena Ramirez-Parra,

Instituto Nacional de Investigación y

Tecnología Agraria y Alimentaria

(INIA), Spain

Qingliang Li,

Moffitt Cancer Center, United States

${ }^{*}$ Correspondence:

Mingyi Bai

baimingyi@sdu.edu.cn

Zhaojun Ding

dingzhaojun@sdu.edu.cn

Huiyu Tian

tianhuiyu@sdu.edu.cn

tThese authors have contributed equally to this work.

Specialty section:

This article was submitted to Plant Physiology,

a section of the journal

Frontiers in Plant Science

Received: 26 October 2017 Accepted: 27 December 2017 Published: 18 January 2018

Citation:

Tian H, Lv B, Ding T, Bai M and Ding Z (2018) Auxin-BR Interaction

Regulates Plant Growth and Development.

Front. Plant Sci. 8:2256.

doi: 10.3389/fpls.2017.02256
Plants develop a high flexibility to alter growth, development, and metabolism to adapt to the ever-changing environments. Multiple signaling pathways are involved in these processes and the molecular pathways to transduce various developmental signals are not linear but are interconnected by a complex network and even feedback mutually to achieve the final outcome. This review will focus on two important plant hormones, auxin and brassinosteroid (BR), based on the most recent progresses about these two hormone regulated plant growth and development in Arabidopsis, and highlight the cross-talks between these two phytohormones.

Keywords: auxin, BR, crosstalk, signaling, plant

\section{INTRODUCTION}

Unlike animals that can move to avoid the adverse surroundings, the sessile plants exhibit a highly developed adaptation to the complicated environmental conditions. To achieve this profound adaptability, communications among cells are necessary. Cell to cell communication in plants involves robust intracellular signaling processing and intricate intercellular signaling networks. Till now there are at least nine signaling substances, named plant hormones, including auxin, brassinosteroid (BR), cytokinin, gibberellins (GA), ethylene, jasmonic acid (JA), strigolactone (SL), abscisic acid (ABA), and salicylic acid (SA) discovered (Druege et al., 2016; Verma et al., 2016). The genetic and physiological studies have revealed the critical roles and functional mechanisms of these above hormones in plant growth and development (Gray, 2004). Based on the previous studies, auxin, BR, GA, SL, and cytokinin mainly function during normal plant growth and development, while $\mathrm{ABA}$, ethylene, JA, and SA play important roles in plant growth response to various biotic and abiotic stresses (Pieterse et al., 2009; Santner et al., 2009; Denance et al., 2013). And also some of these hormones have dual roles, for example, ABA also plays important roles in seed development and dormancy (Seo and Koshiba, 2002). Although each hormone plays predominant roles in certain aspects, many hormones have overlapped activities and the interactions of different hormones control many developmental aspects and growth in response to endogenous developmental and exogenous cues.

Auxin and BR are two major classes of growth-promoting hormones. BR, a group of plantspecific steroid hormones which could interact with other phytohormones such as auxin, cytokinin, ethylene, GA, JA, and SA and regulate a wide range of plant growth and developmental processes including seed germination, cell elongation, vascular differentiation, stomata formation and movement, flowering and male fertility (Saini et al., 2015). Interestingly, each of these processes 
is also controlled by auxin, suggesting these two hormones interplay to control plant development. In this review, we will outline the signal transduction of auxin and BR based on the recent progress and review the crosstalk between auxin and BR mediated plant growth and development.

\section{AUXIN SIGNALING PATHWAY}

Auxin was first recognized as plant hormone because of its role in plant tropism to gravity or light stimuli. Later auxin was chemically identified as indole-3-acetic acid and shown to play essential roles in plethora of plant developmental and physiological processes, including embryogenesis, organogenesis, vascular differentiation, root and shoot development, tropic growth, and fruit development (Estelle, 2011).

Using genetic analysis in Arabidopsis, the molecular mechanism underlying the auxin signal transduction has been well investigated. TRANSPORT INHIBITOR RESPONSE1 (TIR1) was the first identified nuclear receptor of auxin (Ruegger et al., 1998; Dharmasiri et al., 2005). TIR1 encodes a nuclear protein belonging to the F-box protein as a subunit of SCF E3 ubiquitin ligase protein complex (Gray et al., 1999, 2002; Hellmann et al., 2003; Quint et al., 2005), In addition to TIR1, there are three additional F-box proteins namely AUXIN SIGNALING F BOX PROTEINs (AFBs) which show auxinbinding activity and mediate auxin signaling in Arabidopsis (Badescu and Napier, 2006). TIR1 receptor can interact with a group of AUX/IAA (auxin/indole-3-acetic acid) proteins (Dharmasiri et al., 2003). AUX/IAA proteins are negative regulators of auxin signaling and there are 29 members of AUX/IAA encoded in Arabidopsis genome. AUX/IAA proteins could interact with the class of transcriptional regulators, auxin response factors $(\mathrm{ARF})$, to mediate transcriptional responses to auxin. Under high auxin level, AUX/IAA proteins interact with TIR1 as coreceptor of auxin, and can be ubiquitinated by the $\mathrm{SCF}^{\text {tirl }}$ complex and thus be degraded through the ubiquitin-proteasome pathway (Gray et al., 2001; Lanza et al., 2012). Upon the destruction of AUX/IAA repressors, the auxin transcriptional regulators ARFs which include 23 memberships can be released from AUX/IAA repression and thus mediate the auxin responses by activation or repression of target genes (Guilfoyle and Hagen, 2007). The different sets of F-box protein and AUX/IAA or ARFs infer the complexity during auxin signal transduction (Goh et al., 2012; Guilfoyle, 2015; Salehin et al., 2015).

The coordinated action of Aux/IAA transcriptional repressors and ARF transcription factors produces complex gene-regulatory networks which were also reported in Physcomitrella (Lavy et al., 2016). Recently, it was found that CULLIN1 (CUL1) subunit of the SCF interacts with TIR1 and thus regulates TIR1 substrates stability and auxin signaling (Wang et al., 2016). The interaction between TIR1 and Aux/IAA is also influenced by the spatial conformation of Aux/IAAs, controlled by a cyclophilin isomerase LRT2 in rice (Jing et al., 2015). HEAT SHOCK FACTOR 90 (HSP90) and the co-chaperone SGT1, respectively, interacts with TIR1 and thus regulates TIR1 stability, which affects the interactions between TIR1 and Aux/IAA and auxin signaling (Wang et al., 2016).

Besides the TIR1-dependent canonical auxin-signaling pathway, auxin has recently been reported to elicit a diverse range of developmental responses through a non-canonical auxin-signaling mechanism. In this noncanonical auxin sensing process, ARF3/ETTIN controls gene expression through interactions with process-specific transcription factors, which highly enriches auxin-mediated plant developmental diversity (Simonini et al., 2016, 2017).

\section{BR SIGNALING PATHWAY}

BRASSINOSTEROID was first discovered in pollen for its ability to promote cell elongation. Later it was found that BR plays roles in a wide range of plant growth aspects and can respond to biotic and abiotic stresses. Nowadays BR signal transduction pathway was largely clarified by combinations of different methods, including molecular genetics, biochemistry, proteomics, and genomics, etc. The cell-surface kinase BRASSINOSTEROID INSENSITIVE1 (BRI1) was identified as the receptor of BR which can bind to the extracellular domain of BRI1 and activate its kinase activity and thus switch on a signal cascade to regulate transcription (Li and Chory, 1997; Wang et al., 2001; Kinoshita et al., 2005; Kim and Wang, 2010; Clouse, 2011; Hothorn et al., 2011; She et al., 2011; Oh et al., 2012). Upon perception of BR, BRI1 interacts with co-receptor BRI1-ASSOCIATED KINASE 1 (BAK1) and its homolog SOMATIC EMBRYOGENESISRECEPTOR KINASEs (SERKs) to form a more active BR receptor complex (Li et al., 2002; Nam and Li, 2002; Wang et al., 2005; Tang et al., 2008; Gou et al., 2012). Activated BRI1 phosphorylates two substrates of plasma membrane-anchored receptor-like cytoplasmic kinases: BRASSINOSTEROID-SIGNALING KINASES1 (BSK1) and CONSTITUTIVE DIFFERENTIAL GROWTH1 (CDG1) (Tang et al., 2008; Kim et al., 2011), which in turn phosphorylates a PP1-type phosphatase named BRI1-SUPPRESSOR1 (BSU1) to activate BSU1, leading to BSU1 dephosphorylation and inactivation the GSK3-like kinase BRASSINOSTEROID INSENSITIVE2 (BIN2). The kinase activity of BIN2 is also inhibited by HISTONE DEACETYLASE HDA6, which interacts and deacetylates at the K189 of BIN2. When BR levels are low, BRI1 is quiescent due to its negative regulator, BRI1 KINASE INHIBITOR 1 (BKI1) and protein phosphatase 2A (PP2A), while BIN2 phosphorylate two BR homologous transcription factors, BRASSINAZOLE RESISTANT1 (BZR1) and BZR2 (also named BES1 for BRI1-EMS-SUPPRESSOR 1) (He et al., 2002; Wang et al., 2002; Yin et al., 2002; Mora-Garcia et al., 2004; Kim et al., 2009, 2011; Kim and Wang, 2010). When BR levels are high, BIN2 is inactivated, and BZR1 and BZR2 are dephosphorylated by PP2A, and move into nucleus to alter the expression of thousands of BR response genes (He et al., 2005; 
Yin et al., 2005; Sun et al., 2010; Tang et al., 2011; Yu et al., 2011).

\section{THE SYNERGY BETWEEN BR AND AUXIN SIGNALING}

Auxin and BR signal pathways play diverse roles, however, they also showed synergistic and interdependent interactions in a wide range of developmental processes. For example, both auxin and BR signals can promote cell expansion and can interact synergistically to promote hypocotyls elongation (Nemhauser et al., 2004). The response of one of the two pathways in promoting hypocotyl elongation requires the function of the other and the interdependence between BR and auxin pathways (Nemhauser et al., 2004). Auxin increased hypocotyl length in wild-type plants but not in the BR-insensitive mutant bri1-116, and this auxin-insensitive phenotype of bri1-116 was suppressed by the dominant gain-of-function mutant $b z r 1-1 D$, indicating BR or active BZR1 is required for auxin promotion of hypocotyl elongation. It has been found that $\mathrm{BR}$ signaling converges with SUPPRESSOR OF PHYTOCHROME B4-3 (SOB3) to control cell elongation and hypocotyl growth through the regulation of auxin induced SMALL AUXIN UP RNA19 (SAUR19) expression (Favero et al., 2017). On the other hand, the auxin regulated transcription factor SMALL ORGAN SIZE 1 (SMOS1) has recently been found to control cell expansion through the direct interaction with SMOS2/DLT, a member of the GRAS family of transcriptional co-regulators which plays a positive role in $\mathrm{BR}$ signaling in rice (Kim et al., 2009; Tong et al., 2012; Hirano et al., 2017). Auxin related mutants such as iaa3 and arf6/arf8 were less sensitive to BR than was wild-type for hypocotyl elongation, and abolished the hypersensitivity of bzr1-1D to auxin, suggesting the BR and BZR1 promotion of hypocotyl elongation requires ARF6/8. The genome-wide ChIP-Seq analysis revealed that ARF6 shares a vast number of genomic targets (around 50\%) with BZR1 and the light/temperature-regulated transcription factor PIF4 by CHIP-Seq analyses (Oh et al., 2014). BZR1 and PIF4 interact with ARF6 and activate shared target genes by binding to shared target genes cooperatively during hypocotyls elongation (Oh et al., 2014) and many of these overlapping target genes encode cell wall proteins involved in cell expansion.

Brassinosteroid and auxin also play important roles in the maintenance of root apical meristem (RAM) (Durbak et al., 2012). The RAM consists of a small group of rarely dividing cells known as the quiescent center (QC), surrounded by stem cells that give rise to the various toot tissue types. The maintenance of the root stem cell population is regulated by WUSCHELRELATED HOMEOBOX 5 (WOX5) (Sarkar et al., 2007). WOX5 is restricted to the QC by auxin signaling and facilitates proper expression of the PLT genes (Aida et al., 2004; Ding and Friml, 2010). Mutations in the BR receptor gene BRASSINOSTEROID INSENSITIVE 1 (BRI1) result in aberrant cell cycle progression in the RAM and cause a smaller RAMs (Gonzalez-Garcia et al., 2011; Hacham et al., 2011). Auxin is known to stimulate the biosynthesis of BR (Chung et al., 2011), but the activity of
BR does not affect the expression of PIN genes (Hacham et al., 2011). The root tip phenotypes of BR mutants do not show the same as the auxin mutants (Gonzalez-Garcia et al., 2011), indicating that BR act on the RAM independently of auxin.

Brassinosteroid and auxin signals are also synergistically required in the radial pattern formation of vascular bundles (Ibanes et al., 2009). By the combinations of mathematical modeling and biological experiments, auxin maxima, established by asymmetric auxin polar transport, but not changes on auxin levels is important for positioning the vascular bundles. BR signal was shown to serve as a promoting signal for the number of cells in the provascular ring which are consistent with auxin maxima. Thus the establishment of periodic arrangement of vascular bundles in the shoot is under the coordinated action of these two plant hormones (Ibanes et al., 2009). Both signals are also involved in plant root development and the interaction of BR and auxin is mediated by BREVIS RADIX (BRX) during this process. BRX is important for the rate-limiting biosynthesis of $\mathrm{BR}$ and $\mathrm{BR}$ exogenous application can rescue brx mutant defects. Furthermore, auxin-responsive gene expression is globally impaired in brx mutant, and the expression of BRX is strongly induced by auxin and suppressed by BR, implying BR biosynthesis and auxin signaling are connected through a feedback loop involving BRX during root development (Mouchel et al., 2006).

Brassinosteroids and auxin also play synergistic roles during lateral root development. BRs mainly function at the lateral root primordia initiation while auxin is required for both initiation and emergence stages of lateral root formation (Casimiro et al., 2001; Bhalerao et al., 2002; Benkova et al., 2003; Bao et al., 2004). During these processes, BRs increase LRP initiation by promoting acropetal auxin transport in the root but not by affecting endogenous IAA level (Bao et al., 2004). All these reports suggest that the crosstalk between $B R$ and auxin plays an important role in the regulation plant growth and development.

\section{BR REGULATES AUXIN SIGNALING}

Besides the interdependency and cooperation of auxin and BR signals during plant development, BR could mediate auxin signal pathway on multiple levels. BZR1 interacts with ARF proteins to directly target multiple auxin signaling components and genes involved in auxin metabolism such as transport and signaling, including AUX/IAA, PINs, TIR1, and ARFs, etc. (Sun et al., 2010). It was found that Aux/IAA proteins are involved in BR responses and iaa7/axr2-1 and iaa17/axr3-3 mutants showed aberrant BR sensitivity and aberrant BR-induced gene expression in an organ-dependent manner (Nakamura et al., 2006). Exogenous brassinolide (BL) treatment could induce the expression of auxin-responsive genes such as IAA5, IAA19, $I A A 17$, etc., and the expression of the above genes is downregulated in the BR biosynthetic mutant de-etiolated2 (det2), which indicates that functional $\mathrm{BR}$ biosynthesis is partly required for auxin-dependent gene expression (Nakamura et al., 2003; 


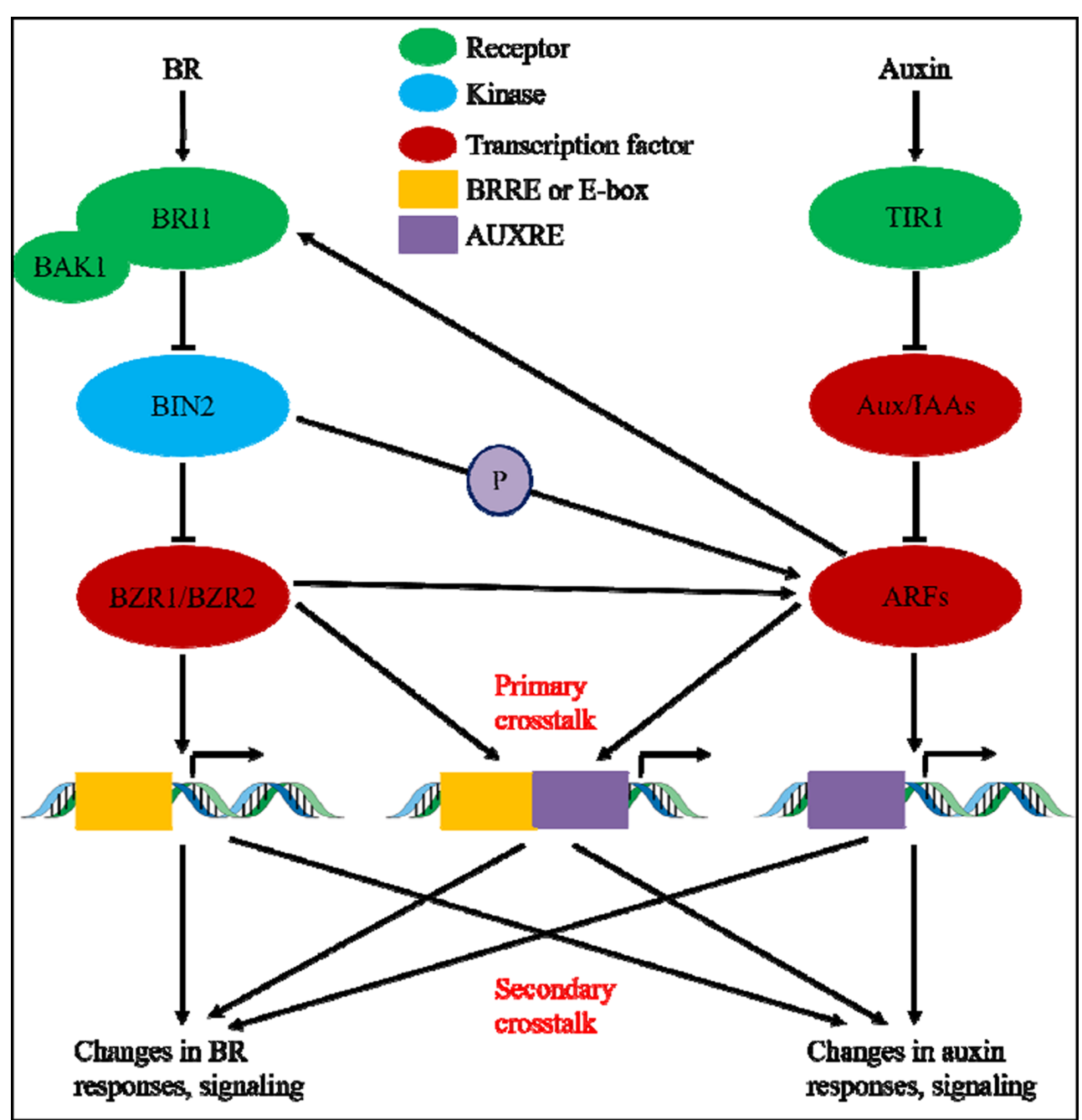

FIGURE 1 | Model of auxin-brassinosteroid (BR) crosstalk. In Arabidopsis, the perceptions of BR and auxin signal are recognized by BRI1 and TIR1 receptors, respectively. BR binds to the extracellular domain of BRI1 and promote it interacts with co-receptor BAK1 to form a more active BR receptor complex, which in turn lead to the dephosphorylation and inactivation of BIN2. The inactivation of BIN2 lead to the dephosphorylation of two BR homologous transcription factors BZR1 and BZR2, which move into nucleus to activate transcription of genes containing BRRE or E-box in their promoter region. BIN2 also can phosphorylate ARF7 and ARF19 to suppress their interaction with AUX/AAs and thereby enhance the transcriptional activity on their target genes. TIR1 receipt the auxin signaling and interact with AUX/IAA proteins as co-receptor of auxin. The AUX/IAA then is degraded through ubiquitin-proteasome pathway, and the auxin transcriptional regulators auxin response factors (ARFs) are released from AUX/IAA repression and activate transcription of genes with auxin responsive elements (AUXRE) in their regulatory region. Some ARFs can also binds to the promoter of BRI 1 and positively regulates its expression which then activates the BR signaling. Primary crosstalk occurs by activation of genes that contain both BRRE/E-box and AUXRE in their promoter region, allowing both signaling pathways to directly regulate transcription. Secondary crosstalk occurs through expression of genes that are either auxin or BR responsive, but the activities of which control expression of genes that regulate the response and signaling of other hormones.

Kim et al., 2006). Additionally, BR also affects auxin flow by regulating the expression of auxin exporters such as PIN4 and PIN7 (Nakamura et al., 2004). During plant gravitropism responses, BRs could enhance the polar accumulation of the auxin exporter PIN2 in the root meristem zone and thus affect the redistribution of auxin from the root tip toward the elongation zones and result in the difference of IAA levels in both upper and lower sides of roots to induce plant gravitropism. During this process, BR activated ROP2 plays an important role in modulating the functional localization of PIN2 through the regulation of the assembly/reassembly of F-actins
(Li et al., 2005). Further studies showed that decreased BL perception and/or concentration could induce CYP79B2, the gene encoding an enzyme converting tryptophan to indole3 -acetaldoxime and thus affect the distribution (Kim et al., 2007).

In addition, it was found that BR signal could regulate auxin signaling output by its negative regulator GSK3 kinase BIN2. The auxin response factor ARF2 was identified as a BIN2 interacting protein in a yeast two-hybrid screen and kinase assay showed BIN2 could phosphorylate ARF2. The phosphorylation of ARF2 results in the loss of its DNA binding ability and repression 
activity of the target genes (Vert et al., 2008). ARF2 is a BZR1 target genes and its expression is reduced by $\mathrm{BR}$ treatment (Sun et al., 2010). Additionally, BIN2 can phosphorylate ARF7 and ARF19 to suppress their interaction with AUX/IAAs and thereby enhance the transcriptional activity on their target genes LATERAL ORGAN BOUNDARIES-DOMAIN16 (LBD16) and LBD29 to regulate lateral root organogenesis (Cho et al., 2014). However, BR plays a minor role during this process and BIN2 is under the control of the TRACHEARY ELEMENT DIFFERENTIATION INHIBITORY FACTOR (TDIF)-TDIF RECEPTOR (TDR) module (Cho et al., 2014). Together, BR can regulate auxin reponses through influencing different auxin signaling components.

\section{AUXIN REGULATES BR SIGNALING}

On the other hand, auxin can also regulate BR signal pathway in certain aspects. The expression of DWARF4, a crucial hydroxylase for BR biosynthesis to control endogenous BR level, is auxin dependent. Auxin treatment could noticeably stimulate the expression of DWARF4 and auxin could inhibit the binding of BZR1 to the promoter of DWARF4. The induction of DWARF4 by auxin requires auxin signaling pathway but not BR signaling pathway (Chung et al., 2011; Yoshimitsu et al., 2011). CPD catalyzing C-3 oxidation of BR was activated by BRX, a putative transcription factor acting downstream of auxin signaling (Mouchel et al., 2006). Further study in rice indicates that exogenous auxin can enhance the transcription expression levels of $\mathrm{BR}$ receptor gene OsBRI1, suggesting that auxin enhances $\mathrm{BR}$ signaling through the regulation of $\mathrm{BR}$ receptors (Sakamoto et al., 2013). Furthermore, the promoter of OsBRI1 possesses an upstream auxin-response element (AuxRE) motif which is targeted by ARF transcription factors. Moreover, mutant studies indicate that upon mutation of AuxRE, the induction of expression of OsBRI1 by auxin is abolished and also the expression of OsBRI1 is down regulated in arf mutant (Sakamoto et al., 2013). It has been reported that OsARF19 binds to the promoter of OsBRI1 and positively regulates its expression which then activates the BR signaling (Zhang et al., 2015). BES1 can bind to the promoter of SMALL AUXINUP RNA 15 (SAUR15) and mediate BR early response gene in Arabidopsis, and this binding could be enhanced by auxin treatment (Walcher and Nemhauser, 2012). Taken together, auxin can also affect BR responses and BR regulated plant growth and development.

\section{CONCLUDING REMARKS AND FUTURE PERSPECTIVE}

During the past nearly four decades, studies on auxinBR pathway interactions have attracted more and more researchers' interest. The appliance of physiological, molecular, genetic, and biochemical tools have greatly deepened our understanding of this issue. Based on the previous studies, $\mathrm{BR}$ and auxin are involved synergistically in multiple plant developmental processes including: hypocotyl elongation, vascular bundles development, root development and tropisms, etc. The interdependency and cooperation of auxin and BR are complicated and involve numerous processes on the molecular level, by sharing the same target genes, regulating each other mutually on multiple levels (Figure 1).

Phosphorylation regulation plays a crucial role in BR signaling pathway, especially during the perception process, $\mathrm{BR}$ is perceived through BRI1 kinase receptor and BAK1 kinase coreceptors, and eventually controls $\mathrm{BR}$ regulated gene expression through influencing downstream transcription factors such as BES1/BZR1 activities (He et al., 2005; Yin et al., 2005; Sun et al., 2010; Tang et al., 2011; Yu et al., 2011). However, ubiquitination regulation seems essential for auxin signaling. Once auxin binds to TIR1 receptor, which acts as an ubiquitin E3-ligase, the activated TIR1 E3-ligase ubiquitinates AUX/IAA proteins, leads to the degradation of these repressors and derepresses ARF transcription factors, and eventually causes auxin regulated gene expression pattern changes and growth responses (Gray et al., 1999, 2002; Hellmann et al., 2003; Quint et al., 2005). Since it has been found that BIN2 kinase, which is well known functioning in BR signaling, could phosphorylate and enhance the activities of ARFs such as ARF2 and ARF7 (Vert et al., 2008; Cho et al., 2014), it will be interesting to test if kinases such as BIN2, which are involved in BR signaling, could also interact with other auxin signaling components such as TIR1 receptor or AUX/IAA repressors, and influence TIR1 E3-ligase activity or AUX/IAA protein stabilities. On the other hand, the role of ubiquitination in BR signaling also needs to be addressed, especially if TIR1 E3-ligase could directly interact with BR signaling components and regulate their protein stabilities.

In addition, using auxin response DR5 and other auxin reporters, it has been observed that auxin regulates plant growth and development in a tissue or cellular dependent manner. The diverse transcriptional outputs depending on the cellular and environmental context (Clark et al., 2014; Etchells et al., 2016; Lavy et al., 2016). Though the spatiotemporal BR signaling has been shown to control root growth through the antagonistic action with auxin (Chaiwanon and Wang, 2015), it is still unknown if the tissue or cellular BR signaling, which could be visualized by pBZR1:BZR1-YFP, is also important to control other processes besides root development. Furthermore, generation of a detailed tissue or cellar map of auxin and BR distributions is currently possible using fluorescence-activated cell sorting or laser microdissection in combination with high-resolution gene expression analysis. This will eventually leads to address if the auxin crosstalks with $\mathrm{BR}$ in a tissue or cellular manner.

\section{AUTHOR CONTRIBUTIONS}

All authors were involved into the writing of this review manuscript. For more information on what constitutes authorship, please refer to our author guidelines. 


\section{FUNDING}

This work is supported by grants from the National Natural Science Foundation of China (Projects 31500229 and

\section{REFERENCES}

Aida, M., Beis, D., Heidstra, R., Willemsen, V., Blilou, I., Galinha, C., et al. (2004). The PLETHORA genes mediate patterning of the Arabidopsis root stem cell niche. Cell 119, 109-120. doi: 10.1016/j.cell.2004.09.018

Badescu, G. O., and Napier, R. M. (2006). Receptors for auxin: will it all end in TIRs? Trends Plant Sci. 11, 217-223.

Bao, F., Shen, J., Brady, S. R., Muday, G. K., Asami, T., and Yang, Z. (2004). Brassinosteroids interact with auxin to promote lateral root development in Arabidopsis. Plant Physiol. 134, 1624-1631. doi: 10.1104/pp.103. 036897

Benkova, E., Michniewicz, M., Sauer, M., Teichmann, T., Seifertova, D., Jurgens, G., et al. (2003). Local, efflux-dependent auxin gradients as a common module for plant organ formation. Cell 115, 591-602. doi: 10.1016/S0092-8674(03) 00924-3

Bhalerao, R. P., Eklof, J., Ljung, K., Marchant, A., Bennett, M., and Sandberg, G. (2002). Shoot-derived auxin is essential for early lateral root emergence in Arabidopsis seedlings. Plant J. 29, 325-332. doi: 10.1046/j.0960-7412.2001. 01217.x

Casimiro, I., Marchant, A., Bhalerao, R. P., Beeckman, T., Dhooge, S., Swarup, R., et al. (2001). Auxin transport promotes Arabidopsis lateral root initiation. Plant Cell 13, 843-852. doi: 10.1105/tpc.13.4.843

Chaiwanon, J., and Wang, Z. Y. (2015). Spatiotemporal brassinosteroid signaling and antagonism with auxin pattern stem cell dynamics in Arabidopsis roots. Curr. Biol. 25, 1031-1042. doi: 10.1016/j.cub.2015.02.046

Cho, H., Ryu, H., Rho, S., Hill, K., Smith, S., Audenaert, D., et al. (2014). A secreted peptide acts on BIN2-mediated phosphorylation of ARFs to potentiate auxin response during lateral root development. Nat. Cell Biol. 16, 66-76. doi: $10.1038 / \mathrm{ncb} 2893$

Chung, Y., Maharjan, P. M., Lee, O., Fujioka, S., Jang, S., Kim, B., et al. (2011). Auxin stimulates DWARF4 expression and brassinosteroid biosynthesis in Arabidopsis. Plant J. 66, 564-578. doi: 10.1111/j.1365-313X.2011.04513.x

Clark, N. M., De Luis Balaguer, M. A., and Sozzani, R. (2014). Experimental data and computational modeling link auxin gradient and development in the Arabidopsis root. Front. Plant Sci. 5:328. doi: 10.3389/fpls.2014.00328

Clouse, S. D. (2011). Brassinosteroid signal transduction: from receptor kinase activation to transcriptional networks regulating plant development. Plant Cell 23, 1219-1230. doi: 10.1105/tpc.111.084475

Denance, N., Sanchez-Vallet, A., Goffner, D., and Molina, A. (2013). Disease resistance or growth: the role of plant hormones in balancing immune responses and fitness costs. Front. Plant Sci. 4:155. doi: 10.3389/fpls.2013.00155

Dharmasiri, N., Dharmasiri, S., and Estelle, M. (2005). The F-box protein TIR1 is an auxin receptor. Nature 435, 441-445. doi: 10.1038/nature03543

Dharmasiri, N., Dharmasiri, S., Jones, A. M., and Estelle, M. (2003). Auxin action in a cell-free system. Curr. Biol. 13, 1418-1422. doi: 10.1016/S0960-9822(03) 00536-0

Ding, Z., and Friml, J. (2010). Auxin regulates distal stem cell differentiation in Arabidopsis roots. Proc. Natl. Acad. Sci. U.S.A. 107, 12046-12051. doi: 10.1073/ pnas. 1000672107

Druege, U., Franken, P., and Hajirezaei, M. R. (2016). Plant hormone homeostasis, signaling, and function during adventitious root formation in cuttings. Front. Plant Sci. 7:381. doi: 10.3389/fpls.2016.00381

Durbak, A., Yao, H., and Mcsteen, P. (2012). Hormone signaling in plant development. Curr. Opin. Plant Biol. 15, 92-96. doi: 10.1016/j.pbi.2011.12.004

Estelle, M. (2011). Auxin Signaling: From Synthesis to Systems Biology; A Subject Collection from Cold Spring Harbor Perspectives in Biology. Cold Spring Harbor, NY: Cold Spring Harbor Laboratory Press.

Etchells, J. P., Smit, M. E., Gaudinier, A., Williams, C. J., and Brady, S. M. (2016). A brief history of the TDIF-PXY signalling module: balancing meristem identity and differentiation during vascular development. New Phytol. 209, 474-484. doi: $10.1111 /$ nph. 13642
31770305), Shandong Province Natural Science Foundation of Major Basic Research Program (2017C03), Shandong Provincial Funds for Distinguished Young Scholars (2014JQ20 1408).

Favero, D. S., Le, K. N., and Neff, M. M. (2017). Brassinosteroid signaling converges with SUPPRESSOR OF PHYTOCHROME B4-\#3 to influence the expression of SMALL AUXIN UP RNA genes and hypocotyl growth. Plant J. 89, 1133-1145. doi: 10.1111/tpj.13451

Goh, T., Joi, S., Mimura, T., and Fukaki, H. (2012). The establishment of asymmetry in Arabidopsis lateral root founder cells is regulated by LBD16/ASL18 and related LBD/ASL proteins. Development 139, 883-893. doi: 10.1242/dev.07 1928

González-García, M. P., Vilarrasa-Blasi, J., Zhiponova, M., Divol, F., MoraGarcía, S., Russinova, E., et al. (2011). Brassinosteroids control meristem size by promoting cell cycle progression in Arabidopsis roots. Development 138, 849-859. doi: 10.1242/dev.057331

Gou, X., Yin, H., He, K., Du, J., Yi, J., Xu, S., et al. (2012). Genetic evidence for an indispensable role of somatic embryogenesis receptor kinases in brassinosteroid signaling. PLOS Genet. 8:e1002452. doi: 10.1371/journal.pgen.1002452

Gray, W. M. (2004). Hormonal regulation of plant growth and development. PLOS Biol. 2:e311. doi: 10.1371/journal.pbio.0020311

Gray, W. M., Del Pozo, J. C., Walker, L., Hobbie, L., Risseeuw, E., Banks, T., et al. (1999). Identification of an SCF ubiquitin-ligase complex required for auxin response in Arabidopsis thaliana. Genes Dev. 13, 1678-1691. doi: 10.1101/gad. 13.13.1678

Gray, W. M., Hellmann, H., Dharmasiri, S., and Estelle, M. (2002). Role of the Arabidopsis RING-H2 protein RBX1 in RUB modification and SCF function. Plant Cell 14, 2137-2144. doi: 10.1105/tpc.003178

Gray, W. M., Kepinski, S., Rouse, D., Leyser, O., and Estelle, M. (2001). Auxin regulates SCF(TIR1)-dependent degradation of AUX/IAA proteins. Nature 414, 271-276. doi: 10.1038/35104500

Guilfoyle, T. J. (2015). The PB1 domain in auxin response factor and Aux/IAA proteins: a versatile protein interaction module in the auxin response. Plant Cell 27, 33-43. doi: 10.1105/tpc.114.132753

Guilfoyle, T. J., and Hagen, G. (2007). Auxin response factors. Curr. Opin. Plant Biol. 10, 453-460. doi: 10.1016/j.pbi.2007.08.014

Hacham, Y., Holland, N., Butterfield, C., Ubeda-Tomas, S., Bennett, M. J., Chory, J., et al. (2011). Brassinosteroid perception in the epidermis controls root meristem size. Development 138, 839-848. doi: 10.1242/dev.061804

He, J. X., Gendron, J. M., Sun, Y., Gampala, S. S., Gendron, N., Sun, C. Q., et al. (2005). BZR1 is a transcriptional repressor with dual roles in brassinosteroid homeostasis and growth responses. Science 307, 1634-1638. doi: 10.1126/ science. 1107580

He, J. X., Gendron, J. M., Yang, Y., Li, J., and Wang, Z. Y. (2002). The GSK3-like kinase BIN2 phosphorylates and destabilizes BZR1, a positive regulator of the brassinosteroid signaling pathway in Arabidopsis. Proc. Natl. Acad. Sci. U.S.A. 99, 10185-10190. doi: 10.1073/pnas.152342599

Hellmann, H., Hobbie, L., Chapman, A., Dharmasiri, S., Dharmasiri, N., Del Pozo, C., et al. (2003). Arabidopsis AXR6 encodes CUL1 implicating SCF E3 ligases in auxin regulation of embryogenesis. EMBO J. 22, 3314-3325. doi: $10.1093 / \mathrm{emboj} / \mathrm{cdg} 335$

Hirano, K., Yoshida, H., Aya, K., Kawamura, M., Hayashi, M., Hobo, T., et al. (2017). SMALL ORGAN SIZE 1 and SMALL ORGAN SIZE 2/DWARF and LOW-TILLERING form a complex to integrate auxin and brassinosteroid signaling in rice. Mol. Plant 10, 590-604. doi: 10.1016/j.molp.2016.12.013

Hothorn, M., Belkhadir, Y., Dreux, M., Dabi, T., Noel, J. P., Wilson, I. A., et al. (2011). Structural basis of steroid hormone perception by the receptor kinase BRI1. Nature 474, 467-471. doi: 10.1038/nature10153

Ibanes, M., Fabregas, N., Chory, J., and Cano-Delgado, A. I. (2009). Brassinosteroid signaling and auxin transport are required to establish the periodic pattern of Arabidopsis shoot vascular bundles. Proc. Natl. Acad. Sci. U.S.A. 106, 13630-13635. doi: 10.1073/pnas.0906416106

Jing, H., Yang, X., Zhang, J., Liu, X., Zheng, H., Dong, G., et al. (2015). Peptidylprolyl isomerization targets rice Aux/IAAs for proteasomal degradation during auxin signalling. Nat. Commun. 6:7395. doi: 10.1038/ncomms8395 
Kim, H., Park, P. J., Hwang, H. J., Lee, S. Y., Oh, M. H., and Kim, S. G. (2006). Brassinosteroid signals control expression of the AXR3/IAA17 gene in the cross-talk point with auxin in root development. Biosci. Biotechnol. Biochem. 70, 768-773. doi: $10.1271 /$ bbb.70.768

Kim, T. W., Guan, S., Burlingame, A. L., and Wang, Z. Y. (2011). The CDG1 kinase mediates brassinosteroid signal transduction from BRI1 receptor kinase to BSU1 phosphatase and GSK3-like kinase BIN2. Mol. Cell 43, 561-571. doi: 10.1016/j.molcel.2011.05.037

Kim, T. W., Guan, S., Sun, Y., Deng, Z., Tang, W., Shang, J. X., et al. (2009). Brassinosteroid signal transduction from cell-surface receptor kinases to nuclear transcription factors. Nat. Cell Biol. 11, 1254-1260. doi: 10.1038/ ncb1970

Kim, T. W., Lee, S. M., Joo, S. H., Yun, H. S., Lee, Y., Kaufman, P. B., et al. (2007). Elongation and gravitropic responses of Arabidopsis roots are regulated by brassinolide and IAA. Plant Cell Environ. 30, 679-689. doi: 10.1111/j.13653040.2007.01659.x

Kim, T. W., and Wang, Z. Y. (2010). Brassinosteroid signal transduction from receptor kinases to transcription factors. Annu. Rev. Plant Biol. 61, 681-704. doi: 10.1146/annurev.arplant.043008.092057

Kinoshita, T., Cano-Delgado, A., Seto, H., Hiranuma, S., Fujioka, S., Yoshida, S., et al. (2005). Binding of brassinosteroids to the extracellular domain of plant receptor kinase BRI1. Nature 433, 167-171. doi: 10.1038/nature03227

Lanza, M., Garcia-Ponce, B., Castrillo, G., Catarecha, P., Sauer, M., RodriguezSerrano, M., et al. (2012). Role of actin cytoskeleton in brassinosteroid signaling and in its integration with the auxin response in plants. Dev. Cell 22, 1275-1285. doi: 10.1016/j.devcel.2012.04.008

Lavy, M., Prigge, M. J., Tao, S., Shain, S., Kuo, A., Kirchsteiger, K., et al. (2016). Constitutive auxin response in Physcomitrella reveals complex interactions between Aux/IAA and ARF proteins. eLife 5:e13325. doi: 10.7554/eLife. 13325

Li, J., and Chory, J. (1997). A putative leucine-rich repeat receptor kinase involved in brassinosteroid signal transduction. Cell 90, 929-938. doi: 10.1016/S00928674(00)80357-8

Li, J., Wen, J., Lease, K. A., Doke, J. T., Tax, F. E., and Walker, J. C. (2002). BAK1, an Arabidopsis LRR receptor-like protein kinase, interacts with BRI1 and modulates brassinosteroid signaling. Cell 110, 213-222. doi: 10.1016/S00928674(02)00812-7

Li, L., Xu, J., Xu, Z. H., and Xue, H. W. (2005). Brassinosteroids stimulate plant tropisms through modulation of polar auxin transport in Brassica and Arabidopsis. Plant Cell 17, 2738-2753. doi: 10.1105/tpc.105.034397

Mora-Garcia, S., Vert, G., Yin, Y., Cano-Delgado, A., Cheong, H., and Chory, J. (2004). Nuclear protein phosphatases with Kelch-repeat domains modulate the response to brassinosteroids in Arabidopsis. Genes Dev. 18, 448-460. doi: $10.1101 /$ gad.1174204

Mouchel, C. F., Osmont, K. S., and Hardtke, C. S. (2006). BRX mediates feedback between brassinosteroid levels and auxin signalling in root growth. Nature 443, 458-461. doi: 10.1038/nature05130

Nakamura, A., Goda, H., Shimada, Y., and Yoshida, S. (2004). Brassinosteroid selectively regulates PIN gene expression in Arabidopsis. Biosci. Biotechnol. Biochem. 68, 952-954. doi: 10.1271/bbb.68.952

Nakamura, A., Higuchi, K., Goda, H., Fujiwara, M. T., Sawa, S., Koshiba, T., et al. (2003). Brassinolide induces IAA5, IAA19, and DR5, a synthetic auxin response element in Arabidopsis, implying a cross talk point of brassinosteroid and auxin signaling. Plant Physiol. 133, 1843-1853. doi: 10.1104/pp.103.030031

Nakamura, A., Nakajima, N., Goda, H., Shimada, Y., Hayashi, K., Nozaki, H., et al. (2006). Arabidopsis $A u x / I A A$ genes are involved in brassinosteroid-mediated growth responses in a manner dependent on organ type. Plant J. 45, 193-205. doi: 10.1111/j.1365-313X.2005.02582.x

Nam, K. H., and Li, J. (2002). BRI1/BAK1, a receptor kinase pair mediating brassinosteroid signaling. Cell 110, 203-212. doi: 10.1016/S0092-8674(02) 00814-0

Nemhauser, J. L., Mockler, T. C., and Chory, J. (2004). Interdependency of brassinosteroid and auxin signaling in Arabidopsis. PLOS Biol. 2:E258. doi: 10.1371/journal.pbio.0020258

Oh, E., Zhu, J. Y., Bai, M. Y., Arenhart, R. A., Sun, Y., and Wang, Z. Y. (2014). Cell elongation is regulated through a central circuit of interacting transcription factors in the Arabidopsis hypocotyl. eLife 3:e03031. doi: 10.7554/eLife. 03031
Oh, M. H., Wang, X., Clouse, S. D., and Huber, S. C. (2012). Deactivation of the Arabidopsis BRASSINOSTEROID INSENSITIVE 1 (BRI1) receptor kinase by autophosphorylation within the glycine-rich loop. Proc. Natl. Acad. Sci. U.S.A. 109, 327-332. doi: 10.1073/pnas.1108321109

Pieterse, C. M., Leon-Reyes, A., Van Der Ent, S., and Van Wees, S. C. (2009). Networking by small-molecule hormones in plant immunity. Nat. Chem. Biol. 5, 308-316. doi: $10.1038 /$ nchembio. 164

Quint, M., Ito, H., Zhang, W., and Gray, W. M. (2005). Characterization of a novel temperature-sensitive allele of the CUL1/AXR6 subunit of SCF ubiquitinligases. Plant J. 43, 371-383. doi: 10.1111/j.1365-313X.2005.02449.x

Ruegger, M., Dewey, E., Gray, W. M., Hobbie, L., Turner, J., and Estelle, M. (1998). The TIR1 protein of Arabidopsis functions in auxin response and is related to human SKP2 and yeast grrlp. Genes Dev. 12, 198-207. doi: 10.1101/gad.12.2.198

Saini, S., Sharma, I., and Pati, P. K. (2015). Versatile roles of brassinosteroid in plants in the context of its homoeostasis, signaling and crosstalks. Front. Plant Sci. 6:950. doi: 10.3389/fpls.2015.00950

Sakamoto, T., Morinaka, Y., Inukai, Y., Kitano, H., and Fujioka, S. (2013). Auxin signal transcription factor regulates expression of the brassinosteroid receptor gene in rice. Plant J. 73, 676-688. doi: 10.1111/tpj.12071

Salehin, M., Bagchi, R., and Estelle, M. (2015). SCFTIR1/AFB-based auxin perception: mechanism and role in plant growth and development. Plant Cell 27, 9-19. doi: 10.1105/tpc.114.133744

Santner, A., Calderon-Villalobos, L. I., and Estelle, M. (2009). Plant hormones are versatile chemical regulators of plant growth. Nat. Chem. Biol. 5, 301-307. doi: $10.1038 /$ nchembio. 165

Sarkar, A. K., Luijten, M., Miyashima, S., Lenhard, M., Hashimoto, T., Nakajima, K., et al. (2007). Conserved factors regulate signalling in Arabidopsis thaliana shoot and root stem cell organizers. Nature 446, 811-814. doi: 10.1038/nature05703

Seo, M., and Koshiba, T. (2002). Complex regulation of ABA biosynthesis in plants. Trends Plant Sci. 7, 41-48. doi: 10.1016/S1360-1385(01)02187-2

She, J., Han, Z., Kim, T. W., Wang, J., Cheng, W., Chang, J., et al. (2011). Structural insight into brassinosteroid perception by BRI1. Nature 474, 472-476. doi: 10.1038/nature10178

Simonini, S., Deb, J., Moubayidin, L., Stephenson, P., Valluru, M., Freire-Rios, A., et al. (2016). A noncanonical auxin-sensing mechanism is required for organ morphogenesis in Arabidopsis. Genes Dev. 30, 2286-2296. doi: 10.1101/gad. 285361.116

Simonini, S., Deb, J., Moubayidin, L., Stephenson, P., Valluru, M., FreireRios, A., et al. (2017). Corrigendum: a noncanonical auxin-sensing mechanism is required for organ morphogenesis in Arabidopsis. Genes Dev. 31:1821. doi: $10.1101 /$ gad.306985.117

Sun, Y., Fan, X. Y., Cao, D. M., Tang, W., He, K., Zhu, J. Y., et al. (2010). Integration of brassinosteroid signal transduction with the transcription network for plant growth regulation in Arabidopsis. Dev. Cell 19, 765-777. doi: 10.1016/j.devcel. 2010.10.010

Tang, W., Kim, T. W., Oses-Prieto, J. A., Sun, Y., Deng, Z., Zhu, S., et al. (2008). BSKs mediate signal transduction from the receptor kinase BRI1 in Arabidopsis. Science 321, 557-560. doi: 10.1126/science. 1156973

Tang, W., Yuan, M., Wang, R., Yang, Y., Wang, C., Oses-Prieto, J. A., et al. (2011). PP2A activates brassinosteroid-responsive gene expression and plant growth by dephosphorylating BZR1. Nat. Cell Biol. 13, 124-131. doi: 10.1038/ncb2151

Tong, H., Liu, L., Jin, Y., Du, L., Yin, Y., Qian, Q., et al. (2012). DWARF AND LOW-TILLERING acts as a direct downstream target of a GSK3/SHAGGY-like kinase to mediate brassinosteroid responses in rice. Plant Cell 24, 2562-2577. doi: 10.1105/tpc.112.097394

Verma, V., Ravindran, P., and Kumar, P. P. (2016). Plant hormone-mediated regulation of stress responses. BMC Plant Biol. 16:86. doi: 10.1186/s12870-0160771-y

Vert, G., Walcher, C. L., Chory, J., and Nemhauser, J. L. (2008). Integration of auxin and brassinosteroid pathways by Auxin Response Factor 2. Proc. Natl. Acad. Sci. U.S.A. 105, 9829-9834. doi: 10.1073/pnas.0803996105

Walcher, C. L., and Nemhauser, J. L. (2012). Bipartite promoter element required for auxin response. Plant Physiol. 158, 273-282. doi: 10.1104/pp.111.187559

Wang, R., Zhang, Y., Kieffer, M., Yu, H., Kepinski, S., and Estelle, M. (2016). HSP90 regulates temperature-dependent seedling growth in Arabidopsis by stabilizing the auxin co-receptor F-box protein TIR1. Nat. Commun. 7:10269. doi: $10.1038 /$ ncomms 10269 
Wang, X., Li, X., Meisenhelder, J., Hunter, T., Yoshida, S., Asami, T., et al. (2005). Autoregulation and homodimerization are involved in the activation of the plant steroid receptor BRI1. Dev. Cell 8, 855-865. doi: 10.1016/j.devcel.2005. 05.001

Wang, Z. Y., Nakano, T., Gendron, J., He, J., Chen, M., Vafeados, D., et al. (2002). Nuclear-localized BZR1 mediates brassinosteroid-induced growth and feedback suppression of brassinosteroid biosynthesis. Dev. Cell 2, 505-513. doi: 10.1016/S1534-5807(02)00153-3

Wang, Z. Y., Seto, H., Fujioka, S., Yoshida, S., and Chory, J. (2001). BRI1 is a critical component of a plasma-membrane receptor for plant steroids. Nature 410, 380-383. doi: 10.1038/35066597

Yin, Y., Vafeados, D., Tao, Y., Yoshida, S., Asami, T., and Chory, J. (2005). A new class of transcription factors mediates brassinosteroid-regulated gene expression in Arabidopsis. Cell 120, 249-259. doi: 10.1016/j.cell.2004.11.044

Yin, Y., Wang, Z. Y., Mora-Garcia, S., Li, J., Yoshida, S., Asami, T., et al. (2002). BES1 accumulates in the nucleus in response to brassinosteroids to regulate gene expression and promote stem elongation. Cell 109, 181-191. doi: 10.1016/ S0092-8674(02)00721-3

Yoshimitsu, Y., Tanaka, K., Fukuda, W., Asami, T., Yoshida, S., Hayashi, K., et al. (2011). Transcription of DWARF4 plays a crucial role in auxinregulated root elongation in addition to brassinosteroid homeostasis in
Arabidopsis thaliana. PLOS ONE 6:e23851. doi: 10.1371/journal.pone.00 23851

Yu, X., Li, L., Zola, J., Aluru, M., Ye, H., Foudree, A., et al. (2011). A brassinosteroid transcriptional network revealed by genome-wide identification of BESI target genes in Arabidopsis thaliana. Plant J. 65, 634-646. doi: 10.1111/j.1365-313X. 2010.04449.x

Zhang, S., Wang, S., Xu, Y., Yu, C., Shen, C., Qian, Q., et al. (2015). The auxin response factor, OsARF19, controls rice leaf angles through positively regulating OsGH3-5 and OsBRI1. Plant Cell Environ. 38, 638-654. doi: 10.1111/pce. 12397

Conflict of Interest Statement: The authors declare that the research was conducted in the absence of any commercial or financial relationships that could be construed as a potential conflict of interest.

Copyright (c) 2018 Tian, Lv, Ding, Bai and Ding. This is an open-access article distributed under the terms of the Creative Commons Attribution License (CC BY). The use, distribution or reproduction in other forums is permitted, provided the original author(s) or licensor are credited and that the original publication in this journal is cited, in accordance with accepted academic practice. No use, distribution or reproduction is permitted which does not comply with these terms. 